\title{
Isolamento de Mierosporum cookei do pêlo de animais silvestres capturados na regiào amazônica
}

\author{
Mário A. P. Moraes (") \\ Margarida M. R. Almeida (")
}

\begin{abstract}
Resumo
E referido o achado, pela primeira vez na regiāo amazônica, de Microsporum cookei, fungo dermatófico geofílico cuja existência só fora registrada, até agora, no Estado de São Paulo. Seu isolamento fez-se a partir de pêlos de animais silvestres capturados na localidade de Mojuí dos Campos, perto da cidade de Santarém, Estado do Pará. Dentre 43 animais testados, 10 abrigavam o fungo no pêlo, sem qualquer lesão cutânea aparente. Além da forma assexuada de M. cookei, foi também obtida sua forma sexuada ou perfeita (Nannizzia cajetani).
\end{abstract}

\section{INTRODUÇÃo}

Microsporum cookei, um dermatófito ligado ao solo, foi descrito por Ajello (1959), que o isolou principalmente do pêlo de animais silvestres. O mesmo autor (1961) descreveu também Nannizzia cajetani, a forma sexuada do fungo.

Parece ser bastante larga a distribuição desta espécie. Embora não referido muitas vezes, já se demonstrou a existência de $M$. cookei, no solo ou em animais, em todos os continentes.

Considerado a princípio como não patogênico (todas as tentativas de inoculação experimental feitas por Ajello, 1959, resultaram negativas), casos esporádicos, em que foi possível isolar-se o fungo de lesões na pele, tanto em humanos (Balabanoff, 1965; Schick, 1966; Jaksch \& Thurner, 1965; Lundell, 1969; Frey, 1971) como em animais (Kaplan, ref. por Ajello, 1959; Jaksch \& Thurner, 1965; Lundell, 1969), mostraram que em determinadas condições pode $M$. cookei transformar-se em parasita. As Infecções por ele determinadas no homem são em geral leves e curam, espontaneamente, ao cabo de algumas semanas (Lundell, 1969; Frey, 1971). Não deixa de ser curioso que em dois dos poucos casos humanos conhecidos houvesse associação com animais portadores de lesões - em um caso com um gato (Jaksch \& Thurner, 1965) e no outro com um cão (Lundell, 1969) - das quais também se isolou o fungo.

Apenas duas vezes foi $M$. cookei assinalado no Brasil, ambas no Estado de São Paulo. Castro \& Alchorne (1962), semeando diretamente em Sabouraud-glicose pêlos de 31 animais capturados em duas localidades do interior daquele Estado, conseguiram isolar 4 cepas, provenientes de três roedores dos gêneros Akodon, Nectomys e Oxymycterus. Rogers \& Beneke (1964), de 10 amostras de solo coletadas em duas áreas da praia de Santos. no litoral de São Paulo, obtiveram duas positivas para $M$. cookei. Apesar de terem examinado mais 92 amostras de terra, colhidas em Belo Horizonte, São Paulo, Piracicaba e localidades vizinhas, somente as duas mencionadas. da praia de Santos, mostraram-se positivas.

Em 1975, por ocasião de uma epidemia causada pelo vírus Oropouche, no Município de Santarém, Estado do Pará, capturaram-se, em busca de um possivel reservatório, no local onde mais intensa era a epidemia, diversos animais silvestres, cujos pêlos foram aproveltados, como de praxe, para a pesquisa de ceratinófilos. Dentre os fungos deste grupo que puderam ser isolados figurava M. cookei. Anteriormente, centenas de amostras semelhantes, bem como de terra, colhidas nos mais diversos pontos da Amazônia brasileira (Moraes, dados inéditos), já haviam sido por nós testadas, sem nunca ter ele aparecido entre

(*) - Instituto Evandro Chagas - Fundação Serviços de Saúdé Pública (FSESP). Belém, Pará. 
os dermatófitos isolados. Por outro lado, nos raros trabalhos sobre o assunto feitos no Norte do Brasil (Fonseca, 1976; Castrillón et al., 1976), não há referência ao seu achado. Esta é, portanto, a primeira vez que $M$. cookei tem sua existência assinalada na região.

\section{MATERIAL E MÉTODOS}

Os animais silvestres, de cujo pelo foi M. cookei isolado, provinham dos arredores de Mojui dos Campos, pequena vila situada a cerca de $20 \mathrm{~km}$ ao sul da cidade de Santarém, Estado do Pará. De cada animal, após o sacrifício, punhados de pêlos eram retirados dos flancos e do dorso, por meio de pinças, sendo as amostras guardadas em papel esterilizado até o momento de seu emprego.

O método usado no isolamento foi seme Ihante ao de Vanbreuseghem (1952), para obtenção de dermatófitos do solo: cada amostra era colocada em uma placa de Petri, de 6 $\mathrm{cm}$ de diâmetro, em cujo fundo havia uma delgada camada de terra esterilizada; procuravase distribuir de modo uniforme os pêlos sobre a terra, e esta, por fim, era umedecida com algumas gotas de água destilada estéril. A intervalos de uma semana, durante um período de 2 meses, examinavam-se as placas para verificar qualquer crescimento miceliano sobre os pelos. Quando isto ocorria, transferia-se para tubos com meio de Sabouraud-glicose uma pequena porção dos pêlos infectados ou, simplesmente, do micélio em crescimento. Depois de uma semana, fragmentos das colônias porventura surgidas sobre o meio eram examinadas ao microscópio para reconhecimento dos fungos dermatófitos.

Amostra de 43 animais silvestres foram empregadas neste trabalho. Pertenciam eles aos seguintes gêneros, com o respectivo número de exemplares: Didelphys - 19; Proechimys - 8; Caluromys - 8; Oxymycterus 4; Zigodontomys - 3; e Orizomys - 1 .

\section{Resultados}

Em 10 das 43 amostras testadas houve crescimentō de Microsporum cookei. A forma

Vários outros dermatófitos - cuja relação vai a seguir, com o número de placas positivas

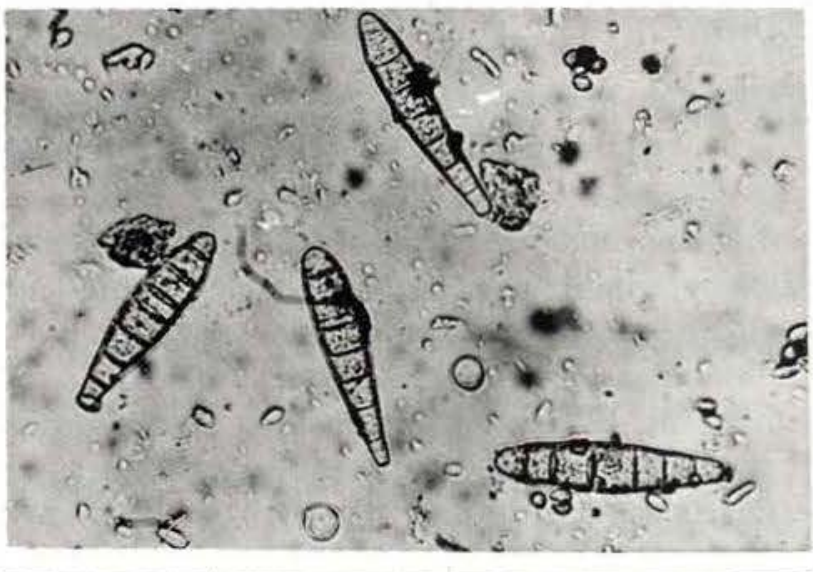

'Foto 1 - Macroconídios jovens de M. cookei, desenvolvidos sobre os pêlos. Notar a forma em clava

perfeita (Nannizzia cajetani) apareceu 4 vezes. Os animais dos quais as amostras positivas foram obtidas pertenciam aos gêneros seguintes, com o número respectivo de exemplares: Didelphys - 4; Proechimys - 2; Zigodontomys -2 ; Caluromys -1 ; e Oxymycterus -1 .

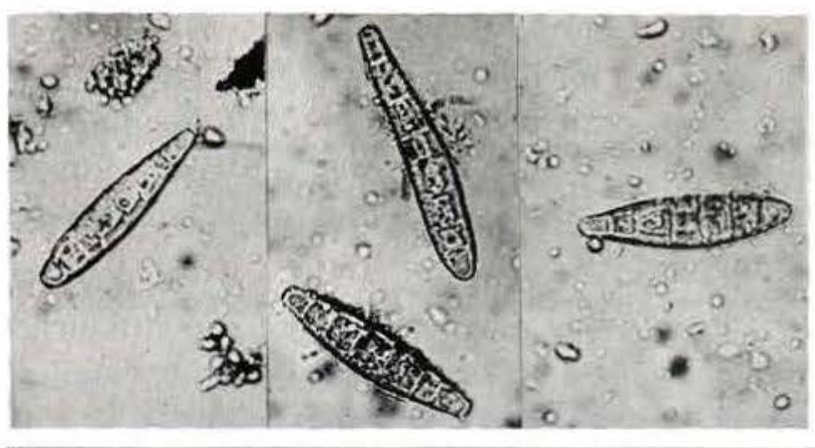

Foto 2 - Macroconídios de M. cookei com sua parede espessa caracteristica

- foram também isolados das 43 amostras, alguns junto com $M$. cookei: Trichophyton terrestre - 11; Chrysosporium indicum - 4; Chrysosporium tropicum - 2; Chrysosporium keratinophilum - 1; e Arthroderma tuberculalum -1 .

\section{COMEnTÁrios}

Muitos dermatófitos, dentre os quais uma espécie nova (Moraes et al., 1967) - Microsporum amazonicum - já foram isolados do solo e do pêlo de animais silvestres na região amazônica. Alguns têm uma distribuição larga, 
como T. terrestre, C. indicum, C. tropicum, M. racemosum e $A$. tuberculatum, enquanto outros apresentam uma distribuição bem restrita, como parece ser o caso de M. cookei. Em uma série de inquéritos que já abrange grande parte da Amazônia brasileira, somente na localidade de Mojuí dos Campos foi ele até agora encontrado. A existência no solo de um fator necessário ao desenvolvimento do fungo talvez pudesse explicar tal fato.

\section{AGRADECIMENTOS}

Os autores agradecem ao Dr. Libero Ajello, do Center for Disease Control, Atlanta, Georgia, por ter confirmado a identificação de M. cookei.

\section{SUMMARY}

Microsporum cookei, a geophilic dermatophyte, was isolated from the hairs of wild animals captured at Mojui dos Campos, a small village near the city of Santarém, State of Pará. This is the first time that this fungus has been found in the Amazon Region. 10 out of 43 animals examined for dermatophytes, all of them free of skin lesions, dad spores of the fungus in the fur. The dermatophyte was also obtained in its sexual form (Nannizia cajetani).

\section{BIBLIOGRAFIA CITADA}

\section{AJELL, L.}

$$
\begin{aligned}
& 1959 \text { - A new Microsporum and its occurence in } \\
& \text { soil and animals. Mycologia, } 51: 69-76 \text {. } \\
& 1961 \text { - The ascigerous state of Microsporum cookei. } \\
& \text { Sabourraudia, 1: 173-177. }
\end{aligned}
$$

BALABANOFF, V. A.
1965 - Dimorphism of dermatophytes with regard to the grade of parasitic adaption and their classification. Mycopathologia et My- cologia Applicata, 25 : 323-329.

Castrillón, A. L.; Moraes, M. A. P. \& Furtado, M. S. S.

1976 - Isolamento de Microsporum amazonicum do solo do Estado do Amazonas, Brasil. Acta Amazonica, 6 : 487-490.

Gastro, R. M. \& Alchorne, M. M. A.

1962 - Microsporum cookei; seu isolamento de roedores silvestres brasileiros. Revista do Instituto de Medicina Tropical de São Paulo, 4: 35-37,

Fonseca, O. J. M.

1976 - Fungos ceratinofílicos no solo de Manaus. Acta Amazonica, $6:$ 63-65.

FREY, D.

1971 - Isolation of Microsporum cookei from a human case. Sabouraudia, 9: 146-148.

JAKSCH, W, U, \& THURNER, J.

1965 - Durch Microsporum cookei verursachte Dermatomykose eines Kindes und seiner Katze. Mykosen Tagung, Munchen.

LUNDELL, E.

1969 - Microsporum cookei Ajello in an eczematous skin lesion. Mykosen, 12: 123-126.

Moraes, M.; Borelli, D. \& Feo, M.

1967 - Microsporum amazonicum nova species. Medicina Cutanea, Barcelona, 2 : 201-205.

Rogers, A. L. \& BeneKe, E. S.

1964 - Human pathogenic fungi recovered from Brazilian soil. Mycopathologia et Mycologia Applicata, $22:$ 15-20.

ScHICK, G. W.

$$
\begin{aligned}
& 1966 \text { - Drei positive Befunde von Microsporum } \\
& \text { cookei Ajello } 1959 \text { in Hautlesionen beim } \\
& \text { Menschen. Dermatologische Wochenschrift, } \\
& \text { 152: 177-184. }
\end{aligned}
$$

VANBREUSEGHEM, $\mathrm{R}$.
$19 \$ 2$ - Technique biologique pour l'isolement des dermatophytes du sol. Ann. Soc. Belge Méd. trop., 32 : 173-178.

(Aceito para publicação em 20/01/78) 